\title{
A Model-Reference Sliding Mode for Dual-Stage Actuator Servo Control in HDD
}

\author{
S. Sonkham, U. Pinsopon, W. Chatlatanagulchai
}

\begin{abstract}
This paper presents a method of sliding mode control (SMC) designing and developing for the servo system in a dual-stage actuator (DSA) hard disk drive. Mathematical modeling of hard disk drive actuators is obtained, extracted from measuring frequency response of the voice-coil motor (VCM) and PZT micro-actuator separately. Matlab software tools are used for mathematical model estimation and also for controller design and simulation. A modelreference approach for tracking requirement is selected as a proposed technique. The simulation results show that performance of a modelreference SMC controller design in DSA servo control can be satisfied in the tracking error, as well as keeping the positioning of the head within the boundary of $+/-5 \%$ of track width under the presence of internal and external disturbance. The overall results of model-reference SMC design in DSA are met per requirement specifications and significant reduction in \%off track is found when compared to the single-state actuator (SSA).
\end{abstract}

Keywords-Hard Disk Drive, Dual-Stage Actuator, Track Following, HDD Servo Control, Sliding Mode Control, ModelReference, Tracking Control.

\section{INTRODUCTION}

$\mathrm{I}^{\mathrm{N}}$ Hard Disk Drive (HDD) industry, capacity and access performance are major product specifications. To achieve larger capacity, increasing areal density is most important. In order to increase areal density in an HDD, a servo system is one of the most significantly factors that need to be improved with high accuracy to achieve the principle requirements. The accuracy of read/write head positioning on the center of a target track is a key factors which requires a positioning in the order of a few nanometers. The conventional HDD used only the VCM is very hard to achieve the requirement because it has several of flexible resonances at high frequencies, which limits the increase of servo bandwidth and the positioning accuracy. In order to develop high bandwidth track-following servo systems, a DSA in the HDD has been proposed as an effective solution to overcome the limitation. DSA has been involved and developed by integrating the piezoelectric materials for acting as a secondary actuator in order to keep the read/write head on the desired track accurately. The suspension-based DSA is being introduced for use in HDDs, by integrating PZT (lead-zirconate-titanate) material placed onto the suspension of head-gimbal assembly (HGA) by locating between the slider head and HGA base plate. In DSA

Siwaphon Sonkham is with King Mongkut's Institute of Technology Ladkrabang, Bangkok, Thailand (e-mail: S2600628@kmitl.ac.th).

Unnat Pinsopon is with King Mongkut's Institute of Technology Ladkrabang, Bangkok, Thailand (e-mail: kpunnat@kmitl.ac.th)

Withit Chatlatanagulchai is with Kasetsart University, Bangkok, Thailand (e-mail: fengwtc@ku.ac.th). servo systems, the VCM is actuated as the primary stage to provide long track seeking with but poor accuracy and slow response time, while the PZT is used as a secondary actuator to provide higher precision and faster response but has limitation in stroke. When combining them with properly designed controllers, both actuators are complementary to each other. The implementation of the HDD servo controller relies on the positioning error signal (PES), which is obtained by reading the position information encoded on the disk's data tracks. The position error is also called track mis-registration (TMR) in the HDD industry. Major TMR sources in the trackfollowing mode includes spindle run out, disk fluttering, bias force, external vibration/shock disturbance, vibrations due to air turbulence, and PES noise [1]-[5].

Various control designs have been developed for DSA servo control and can be largely classified into two categories as: the first group based on decoupled or sequential singleinput single-output (SISO) designs, and the other group based on modern optimal design methodologies such as linear quadratic Gaussian (LQG), loop-transfer recovery (LTR), $\mathrm{H} 2 / \mathrm{H} \infty$ and etc. [6], [7]. In existing literatures, either adaptive or robust control algorithms or both were designed to handle external disturbance and model uncertainties. Hidehiko and Masayoshi (2003) [8] presented a settling control of a DSA system for HDD. The decoupling filter (PZT output estimator) has been discussed and the PZT actuator output is canceled at the VCM controller input. Peng et al. (2004) [9] presented that the DSA can increase the control bandwidth for trackfollowing performance based on a composite nonlinear control approach. Zheng and Tomizuka (2007) [10] proposed an adaptive compensation scheme to estimate and compensate the non-repeatable run out. You and Hong (2007) [11] proposed a robust SMC with a new switching surface for track-following, but not yet in practical. Low and Wong (2007) [12] used a multi-objective genetic algorithm in a controller-tuning scheme for a HDD servo by priority at design objectives rather than the optimization. Taghirad and Jamei (2008) [13] applied an adaptive robust control. Du et al. (2008) [14] devised a linear controller based on solving linear matrix inequalities using an adaptive nonlinear compensation. Horowitz, et al. (2007) [15] studied the vibration compensation for disturbance rejection in DSA. Zhang, et al. (2009) [16] used a Hinf for head positioning and a tracking controlin DSA. The SMC approach has been found for DSA HDD control, Lee, et al. (2000) [17] studied discrete-time design for DSA control using a state-estimator based SMC in the discrete time domain. Wu et al. (2004) [18] developed SMC based learning for track-following in HDD; the learning algorithm utilizes 
shape functions to approximate functions of integral transforms and estimate the control input to reduce repetitive error. Tsang-Li Tai and Jian-Shiang Chen (2005) [19] designed SMC for tracking performances for large and fine range. The settling time could be found by selecting a sliding hyper-plane. Herrmann et al. (2007) [20] studied SMC incorporated into the observer-based micro-actuator. The design state feedback controller used an LQR approach for the discrete-time SMC. Hu et al. (2009) [21] approximated time optimal switching for the time-varying SMC, resulting in smooth transition for both track seeking and following.

This paper presents the design of SMC for DSA trackfollowing servo control in the HDD. The SMC used in this paper is based on the model-reference approach, suitable for tracking of multi-input-multi-output (MIMO) systems, was taken from [22]. The SMC has been studied and proven that it has attributed to robustness for dealing with the parametric uncertainties and disturbances. Basics of the SMC method can be found from [22]-[24] and references therein.DSA plant transfer functions have been obtained by fitting a model from its measuring data that has been extracted by exciting the actuators with the swept frequency sinusoidal input signal. The magnitude and phase from its frequency response data are used in order to obtain an estimated model, and then the proposed design of servo control is developed. Matlab software is used for model estimation, controller design, simulation and evaluation to observe the performance. The organization of this paper is as follows: A description of obtaining the mathematic modeling of dual-stage actuators is provided in Section II. The principles of sliding mode and controller design are given in Section III. Results of this study are shown and discussed in Section IV. Section V. presents conclusions and summarizes the outcome of this work.

\section{Dual Stage Actuator Model}

Normally, the DSA consists of two actuators: the first actuator is the VCM and the second actuator is the PZT so called micro-actuator, which is mounted on the active suspension located between the slider read/write head and HGA base plate. Fig. 1 illustrates the DSA used in designing our control system. $\boldsymbol{u}_{\boldsymbol{v}}$ and $\boldsymbol{u}_{\boldsymbol{p}}$ are the voltage inputs, $\boldsymbol{y}_{\boldsymbol{v}}$ and $\boldsymbol{y}_{\boldsymbol{p}}$ are the displacements of the VCM and PZT respectively.

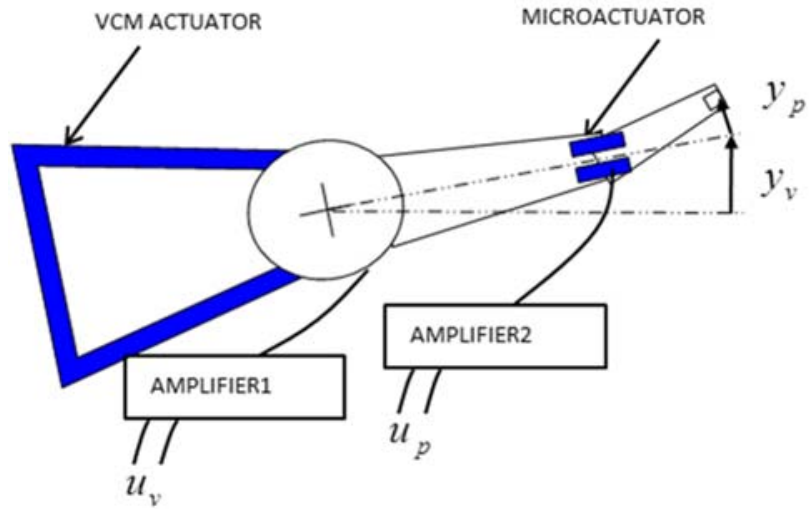

Fig. 1 Schematic of Dual-Stage Actuator in HDD
The frequency responses of the VCM and PZT have been measured from displacement of the head when injecting the sweep sine source at the frequency range of drive operation. Normally, the frequency response of VCM and PZT can be represented by a rigid model $\boldsymbol{G}_{\text {rigid }}$ with their several resonance modes Resas shown in (1) and (2) respectively.

$$
\begin{gathered}
G_{\text {rigid }} \approx \frac{\omega_{p}^{2}}{S^{2}+2 \zeta \omega_{p} S+\omega_{p}^{2}} \\
\operatorname{Re} s \approx \prod_{i=1}^{n} \frac{S^{2}+2 \zeta_{z i} \omega_{z i} S+\omega_{z i}^{2}}{S^{2}+2 \zeta_{p i} \omega_{p i} S+\omega_{p i}^{2}}
\end{gathered}
$$

The model estimation can be obtained by using tools in Matlab software. The comparison plot of frequency response of VCM is shown in Fig. 2 and the response plot of PZT is illustrated in Fig. 3.
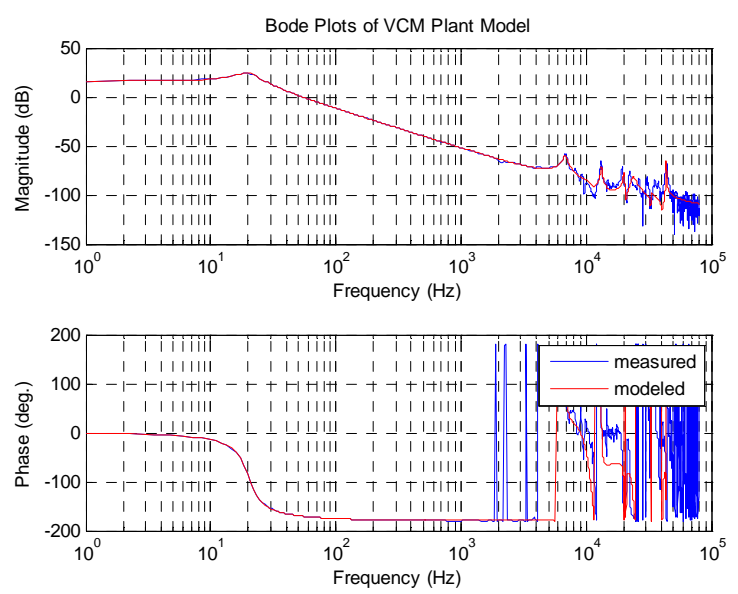

Fig. 2 Comparison of VCM actual and VCM model estimation
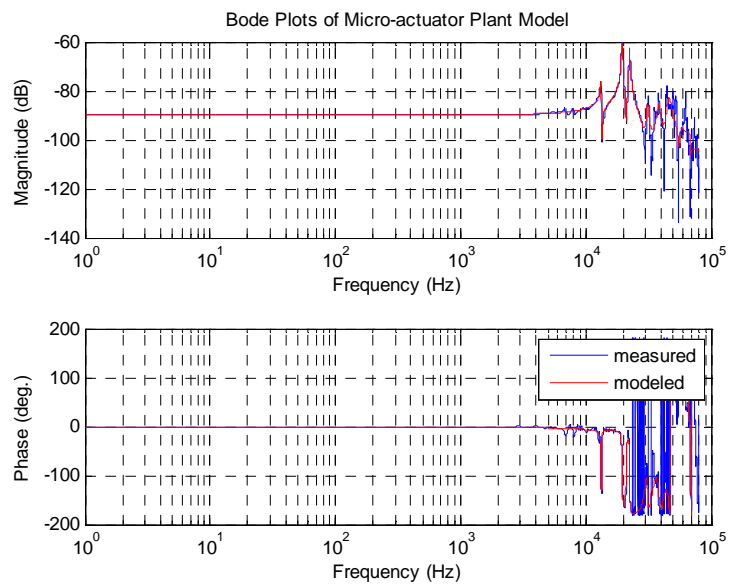

Fig. 3 Comparison of PZT actual plant and PZT model estimation

The state-space model of the second-order rigid model in the LTI system can be obtained in form as below: 


$$
\begin{aligned}
& x(t)^{\prime}=A x(t)+B u(t) \\
& y(t)=C x(t)
\end{aligned}
$$

where $A \in \mathbb{R}^{n x n}, B \in \mathbb{R}^{n x m}$ and $C \in \mathbb{R}^{p x n}, 1 \leq m<n$. The variables $\boldsymbol{x}$ is a vector of state variables, $\boldsymbol{y}$ is a vector of plant outputs, and $\boldsymbol{u}$ is a vector of control. If the rank $(\boldsymbol{B})=m$ and the pair $(\boldsymbol{A}, \boldsymbol{B})$ is controllable, the plant model can be transformed into regular form as

$$
\begin{aligned}
& {\left[\begin{array}{l}
x_{1}{ }^{\prime} \\
x_{2}{ }^{\prime}
\end{array}\right]=\left[\begin{array}{ll}
\mathrm{A}_{11} & \mathrm{~A}_{12} \\
\mathrm{~A}_{21} & \mathrm{~A}_{22}
\end{array}\right]\left[\begin{array}{l}
x_{1} \\
x_{2}
\end{array}\right]+\left[\begin{array}{l}
0 \\
\mathrm{~B}_{2}
\end{array}\right] u} \\
& y=\left[\begin{array}{ll}
\mathrm{C}_{1} & C_{2}
\end{array}\right] x
\end{aligned}
$$

where $\mathrm{X}_{1} \in \mathbb{R}^{n-m}, \mathrm{X}_{2} \in \mathbb{R}^{m}, \boldsymbol{A}_{11}, \boldsymbol{A}_{12}, \boldsymbol{A}_{21}, \boldsymbol{A}_{22}, \boldsymbol{B}_{2}, \boldsymbol{C}_{1}$, and $\boldsymbol{C}_{2}$ are constant matrices with corresponding dimensions. We consider in state-space form so the second-order of the VCM rigid model can be written as below.

$$
\begin{aligned}
& {\left[\begin{array}{l}
x_{1}{ }^{\prime} \\
x_{2}{ }^{\prime}
\end{array}\right]=\left[\begin{array}{ll}
-11.55 & 125.3 \\
-125.3 & -11.55
\end{array}\right] x+\left[\begin{array}{c}
0 \\
36.01
\end{array}\right] u_{v}} \\
& y_{v}=\left[\begin{array}{ll}
23.52 & -0.0979
\end{array}\right] x
\end{aligned}
$$

And then the second-order state-space form of the PZT model can be found as

$$
\begin{aligned}
& {\left[\begin{array}{l}
x_{1}{ }^{\prime} \\
x_{2}{ }^{\prime}
\end{array}\right]=\left[\begin{array}{ll}
-3.167 \times 10^{3} & 1.323 \times 10^{5} \\
-1.323 \times 10^{5} & -3.167 \times 10^{3}
\end{array}\right] x+\left[\begin{array}{c}
0 \\
1.487
\end{array}\right] u_{p}} \\
& y_{p}=\left[\begin{array}{ll}
1.146 & -0.2601] x
\end{array}\right.
\end{aligned}
$$

\section{MODEL REFERENCE SMC DESIGN}

\section{A. Model-References}

The sliding mode control is one of the robust controller designs for nonlinear dynamic plants operating under uncertain conditions. The main advantage of the sliding mode principle is low sensitivity of plant parameter variations, model uncertainties and disturbances. The principle of SMC involves discontinuous control. Usually, SMC is considered a high-speed switching feedback control because of the variable structure control type. The purpose of the switching control law is to drive the plant state trajectory onto a predetermined surface in the state-space and then to maintain the state trajectory on that surface. This surface is called the "sliding surface". The control function is designed in such a way that when the plant state trajectory is "above" the surface, the control drives the plant state "down" to the surface, and when the trajectory is "below" the surface, the control drives the plant state "up" to the surface. As a result, the plant trajectory is always driven toward the sliding surface by the control. The state variables are driven to a sliding surface and maintained there; this process is called "reaching phase". Once, trajectory is intercepted at the sliding surface, the switching control maintains the plant state trajectory on the surface and the state variables on the sliding surface move to the origin, called the "sliding phase". As a result, the closed-loop system dynamics are determined by the plant state trajectory restricted to the sliding surface. In the model-reference SMC, a reference model is relating to the plant output. The SMC controller is designed so that the errors between the reference model state variables and the plant state variables are driven to zeros. As a result, the plant output is close to the reference model output, and then specifications can be met. We consider the reference model as

$$
\begin{aligned}
& w(t)^{\prime}=A_{m} w(t)+B_{m} r(t) \\
& y_{m}(t)=C_{m} w(t)
\end{aligned}
$$

where $A_{m} \in \mathbb{R}^{n x n}, B_{m} \in \mathbb{R}^{n x m}$ and $C_{m} \in \mathbb{R}^{p x n}, 1 \leq m<n$, so the variables $\boldsymbol{w}$ is a vector of state variables, $\boldsymbol{y}_{\boldsymbol{m}}$ is a vector of reference model outputs, and $r$ is a vector of reference inputs. If the $\operatorname{rank}\left(\boldsymbol{B}_{m}\right)=m$ and the pair $\left(\boldsymbol{A}_{m}, \boldsymbol{B}_{m}\right)$ is controllable, the reference model can be transformed into regular form as

$$
\begin{aligned}
& {\left[\begin{array}{l}
w_{1}{ }^{\prime} \\
w_{2}{ }^{\prime}
\end{array}\right]=\left[\begin{array}{ll}
\mathrm{A}_{\mathrm{m} 11} & \mathrm{~A}_{\mathrm{m} 12} \\
\mathrm{~A}_{\mathrm{m} 21} & \mathrm{~A}_{\mathrm{m} 22}
\end{array}\right]\left[\begin{array}{l}
w_{1} \\
w_{2}
\end{array}\right]+\left[\begin{array}{l}
0 \\
\mathrm{~B}_{\mathrm{m} 2}
\end{array}\right] r} \\
& y_{m}=\left[\begin{array}{ll}
\mathrm{C}_{\mathrm{m} 1} & C_{m 2}
\end{array}\right] w
\end{aligned}
$$

where $\mathrm{W}_{1} \in \mathbb{R}^{n-m}, \mathrm{~W}_{2} \in \mathbb{R}^{m}, \boldsymbol{A}_{\boldsymbol{m} 11}, \boldsymbol{A}_{\boldsymbol{m} 12}, \boldsymbol{A}_{\boldsymbol{m} 21}, \boldsymbol{A}_{\boldsymbol{m} 22}, \boldsymbol{B}_{m 2}$, $\boldsymbol{C}_{\boldsymbol{m} 1}$, and $\boldsymbol{C}_{\boldsymbol{m} 2}$ are constant matrices with corresponding dimensions. The reference model $\left(\boldsymbol{A}_{\boldsymbol{m}}, \boldsymbol{B}_{\boldsymbol{m}}\right)$ can be formulated so that the model outputs $\boldsymbol{y}_{\boldsymbol{m}}$ follow the reference inputs $\boldsymbol{r}$ with desirable transient response. One way to find the matrices $A_{m}$ and $\boldsymbol{B}_{\boldsymbol{m}}$ is to use pole-placement method. If we want to design $\boldsymbol{u}$ for the plant model (3) to have desirable closed-loop poles and to ensure steady-state tracking is zero, we choose

$$
\boldsymbol{u}=\boldsymbol{F} \boldsymbol{x}+\boldsymbol{G r}
$$

Then, the closed-loop system becomes $\boldsymbol{x}=(\boldsymbol{A}+\boldsymbol{B} \boldsymbol{F}) \boldsymbol{x}+\boldsymbol{B} \boldsymbol{G r}, \boldsymbol{y}$ $=\boldsymbol{C} \boldsymbol{x}$ and the transfer function from $\boldsymbol{r}$ to $\boldsymbol{y}$ is given by

$$
\begin{aligned}
& \frac{y(s)}{r(s)}=C(s I-(A+B F))^{-1} B G \\
& G=-\left(C(A+B F)^{-1} B\right)^{-1}
\end{aligned}
$$

A full-state feedback matrix $\boldsymbol{F}$ can be designed to place the closed-loop poles at desired locations to achieve desirable transient response. To ensure steady-state tracking of a step reference, using the final-value theorem. Therefore, the reference model can be chosen as $\boldsymbol{A}_{\boldsymbol{m}}=\boldsymbol{A}+\boldsymbol{B} \boldsymbol{F}$ and $\boldsymbol{B}_{\boldsymbol{m}}=\boldsymbol{B} \boldsymbol{G}$. The reference models for both actuators are found by placing the poles of $(\boldsymbol{A}, \boldsymbol{B})$. The poles at $\left[-5 \times 10^{3},-6 \times 10^{3}\right]$ are placed for $\mathrm{VCM}$, then closed-loop reference model can be found as 


$$
\begin{aligned}
& {\left[\begin{array}{l}
w_{m 1}{ }^{\prime} \\
w_{m 2}{ }^{\prime}
\end{array}\right]=\left[\begin{array}{cc}
-11.55 & 125.3 \\
-2.384 \times 10^{5} & -1.099 \times 10^{4}
\end{array}\right] w_{v}+\left[\begin{array}{c}
0 \\
1.018 \times 10^{4}
\end{array}\right] r_{v}} \\
& y_{m v}=\left[\begin{array}{ll}
23.52 & -0.0979
\end{array}\right] w_{v}
\end{aligned}
$$

and the poles $\left[-5 \times 10^{3},-1.1 \times 10^{4}\right]$ are placed to get the reference model of PZT as below.

$$
\begin{aligned}
& {\left[\begin{array}{l}
w_{m 1}{ }^{\prime} \\
w_{m 2}{ }^{\prime}
\end{array}\right]=\left[\begin{array}{cc}
-3.167 \times 10^{3} & 1.323 \times 10^{5} \\
-94.64 & -1.183 \times 10^{4}
\end{array}\right] w_{p}+\left[\begin{array}{c}
0 \\
331.6
\end{array}\right] r_{p}} \\
& y_{m p}=\left[\begin{array}{ll}
1.146 & -0.2601
\end{array}\right] w_{p}
\end{aligned}
$$

\section{B. Sliding Mode Control}

The whole design of an SMC system is to properly choose or design a sliding surface, that the closed-loop system has desired dynamics. And then a switching control that can drive the plant state trajectory onto the sliding surface and maintain it on that surface is to be designed. The control inputs $\boldsymbol{u}$ will make $\boldsymbol{x}$ tracks $\boldsymbol{w}$, corresponding to $\boldsymbol{y}$ tracks $\boldsymbol{y}_{\boldsymbol{m}}$. Let $\boldsymbol{e}=\boldsymbol{x}-\boldsymbol{w}$ be the error between the plant state variables and the reference model state variables. From (3) and (7), the error dynamics are given by

$$
e^{\prime}=A_{m} e+\left(A-A_{m}\right) x+B u-B_{m} r
$$

Consider a control law

$$
u=u_{l}+u_{n}+u_{2}=v+u_{2}
$$

where;

$$
u_{2}=B^{\dagger}\left(A_{m}-A\right) x+B^{\dagger} B_{m} r
$$

$\boldsymbol{u}_{2}$ is an inverse dynamics term with $\boldsymbol{B}^{\dagger}$ as pseudo-inverse of $\boldsymbol{B}$. Substituting (12) into (11), we have $\boldsymbol{e}^{\boldsymbol{\gamma}}=\mathrm{A}_{\mathrm{m}} \boldsymbol{e}+\mathrm{B} \boldsymbol{v}$. Since both plant model and reference model are in regular form, the error dynamics can also be written in regular form as

$$
\begin{aligned}
& e_{1}{ }^{\prime}=A_{m 11} e_{1}+A_{m 12} e_{2} \\
& e_{2}{ }^{\prime}=A_{m 21} e_{1}+A_{m 22} e_{2}+B_{2} v
\end{aligned}
$$

Let the switching surface be

$$
s=S e=S_{1} e_{1}+S_{2} e_{2}=S_{2} M e_{1}+S_{2} e_{2}
$$

where; $\boldsymbol{S}, \boldsymbol{S}_{1}, \boldsymbol{S}_{2}$, and $\boldsymbol{M}$ are constant matrices with appropriate dimensions. $\boldsymbol{S}_{2} \boldsymbol{B}_{2}$ is any nonsingular diagonal matrix, normally chosen to be $\boldsymbol{B}_{2}$ so that $\boldsymbol{S}_{2}=\boldsymbol{I}$. Using a linear change of coordinates

$$
T_{s} \stackrel{\Delta}{=}\left[\begin{array}{cc}
\mathrm{I} & 0 \\
\mathrm{~S}_{1} & \mathrm{~S}_{2}
\end{array}\right], \text { we have }\left[\begin{array}{l}
e_{1} \\
s
\end{array}\right]=T_{s}\left[\begin{array}{l}
e_{1} \\
e_{2}
\end{array}\right]
$$

Choose

$$
u_{l}=-(S B)^{-1}\left(S A_{m}-\Phi S\right) e
$$

where $\Phi$ is any stable design matrix, and then choose

$$
u_{n}=-\rho(S B)^{-1} \frac{P_{2} s}{\left\|P_{2} s\right\|}
$$

where $\rho>0$ is a design parameter and $\mathrm{P}_{2}$ is a symmetric positive definite matrix equation

$$
P_{2} \Phi+\Phi^{T} P_{2}=-I
$$

After a straight-forward derivation, we obtained

$$
\begin{gathered}
e_{1}{ }^{\prime}=\left(A_{m 11} e_{1}-A_{m 12} M\right) e_{1}+\left(A_{m 12} S_{2}{ }^{-1}\right) s \\
s^{\prime}=\Phi s-\rho \frac{P_{2} s}{\left\|P_{2} s\right\|}
\end{gathered}
$$

Consider a Lyapunov function $V_{1}=s^{T} P_{2} s>0$, so the derivative is given by

$$
\begin{aligned}
V_{1}^{\prime} & =s^{T} P_{2} s+s^{T} P_{2} s^{\prime} \\
& \leq\|s\|^{2}-2 \rho\left\|P_{2} s\right\| \\
& \leq-2 \rho \sqrt{\lambda_{\min } P_{2}} \sqrt{V_{1}}
\end{aligned}
$$$$
\leq 0
$$

From the Rayleigh principle, we can conclude that swill approach zeros in finite time and will remain there afterward. Consider another Lyapunov function $V_{2}=e_{1}^{T} P_{1} e_{1}>0$, where $\boldsymbol{P}_{1}$ is a unique symmetric positive definite solution to the Lyapunov equation

$$
P_{1}\left(A_{m 11}-A_{m 12} M\right)+\left(A_{m 11}-A_{m 12} M\right)^{T} P_{1}=-Q_{1},
$$

where $\boldsymbol{Q} \mathbf{1}$ is a design symmetric positive definite matrix and $\boldsymbol{M}$ to be obtained and $\left(\boldsymbol{A}_{\boldsymbol{m} 11}-\boldsymbol{A}_{\boldsymbol{m} 12} \boldsymbol{M}\right)$ is a stable matrix. So that we can conclude that $\boldsymbol{e}_{\boldsymbol{1}}$ will approach zeros in finite time and will remain there afterward, and during time $s=0$, result in $\boldsymbol{e}_{2}$ will also approach zeros.

The advantages of SMC on this approach are as follows:

- Plant model is normally not required. However, knowing the plant model helps improve the quality of the control system, such as utilizing plant models to compute a controller parameters rather than by trial-and-error, and can be feed-forwarded to cancel known terms results in reducing the effort from fast-switching control, therefore reduces chattering. 
- $\quad$ During the sliding phase, the system order reduces from $n$ to $n-m$, where; $n$ is the number of state variables and $m$ is the number of control inputs, making low complexity.

- During the sliding phase, the system is not affected by matched uncertainties, such as disturbances, noise, and plant uncertainties that can be directly altered by the control inputs.

\section{Controller Design}

In this study, the block diagram of the overall control system of DSA is designed as shown in Fig. 4. This is based on the requirements that the micro-actuator must settle down to zero before its next move. During operation, only the total head displacement $\boldsymbol{y}=\boldsymbol{y}_{\boldsymbol{v}}+\boldsymbol{y}_{\boldsymbol{p}}$ is measured. While the displacements for the feedback control system $\boldsymbol{y}_{\boldsymbol{v}}$ and $\boldsymbol{y}_{\boldsymbol{p}}$ were only estimated based on the fact that the micro-actuator can be accurately approximated by a constant gain, under the present range of operating frequency. The objective of SMC designs is to have the displacement of the head tracks a reference input when the following specifications are met:

- The mean of the steady-state error is zero.

- The $3 \sigma$ PES is to be less than $10 \%$ of track width under desired TMR disturbance.

- The 5\% settling time of step response is to be short as short as possible and the overshoot is less than $5 \%$.

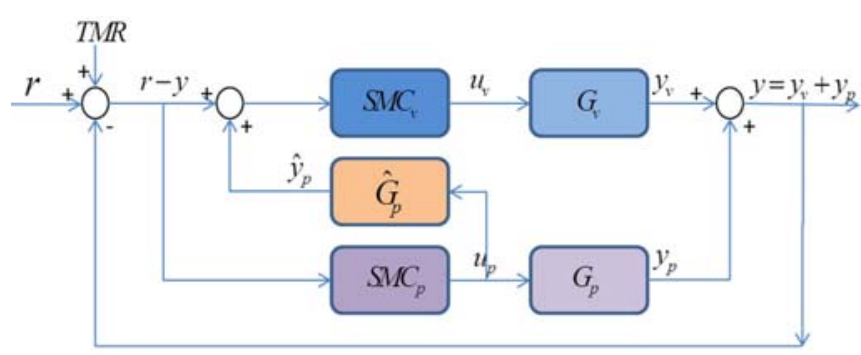

Fig. 4 Schematic diagram of dual-stage actuator control

The DSA control loop from $\boldsymbol{r}$ to $\boldsymbol{y}$ consists of $\boldsymbol{G}_{\boldsymbol{v}}$ is VCM plant that can be considered in a series with desired notch filter for system stabilizing. $\boldsymbol{S M C}$ is a controller designed for VCM plant. $\boldsymbol{G}_{\boldsymbol{p}}$ is plant of PZT that can also be considered to connect in a series with notch filter for improving stability of PZT plant and system. $\boldsymbol{S M C}$ is controller designed of PZT plant. Final block is $\hat{\boldsymbol{G}}_{\boldsymbol{p}}$ decoupling, is estimated as a constant gain of PZT plant, which is placed between the VCM controller input and the PZT controller output. Then, $\hat{y}_{p} \cong y_{p}=y-y_{v}$, so that the PZT actuator output is canceled at the VCM controller input. The $\boldsymbol{r}$ is representing the input commands for DSA servo control to move the head and position onto the target track. Let $\boldsymbol{r}-\boldsymbol{y}$ is to be the controller input of micro-actuator. In the track-following task servo control, ris replaced by TMR to be input to controller. Normally, TMR consists of repeatable run out (RRO), nonrepeatable run out (NRRO) and external disturbances. Considering the controller parameters, choose $\boldsymbol{M}$ to place the poles of (A11, A12) at $[-10,-20]$ and $\boldsymbol{P}_{2}$ to be $\boldsymbol{I}_{\boldsymbol{m}}$, so that $\boldsymbol{S}$ for both actuators will be found as

$$
S_{v}=\left[\begin{array}{ll}
0.0123 & -1
\end{array}\right] \text {, and } S_{p}=\left[\begin{array}{ll}
0.0238 & -1
\end{array}\right]
$$

Then $\Phi$ can be obtained by solving the Lyapunov function in Matlab, and normally set $\rho=1$. The simulation results can be discussed in following section.

\section{Simulation Results}

In the simulation results of the proposed technique, a model-reference SMC for DSA track-following servo control could be satisfied and met most of the specifications. In trackfollowing, the main objective is to achieve the TMR disturbance rejection. The TMR source for this evaluation is referred from [6].

$$
\begin{aligned}
r_{T M R}(t)= & 0.5+0.1 \cos (110 \pi \mathrm{t})+0.05 \sin (220 \pi \mathrm{t}) \\
& +0.02 \sin (440 \pi \mathrm{t})+0.01 \sin (880 \pi \mathrm{t})
\end{aligned}
$$

The results of tracking error comparing between DSA and SSA are shown in Fig. 5, and the $3 \sigma$ of $\%$ off track PES testing are obtained in Fig. 6.
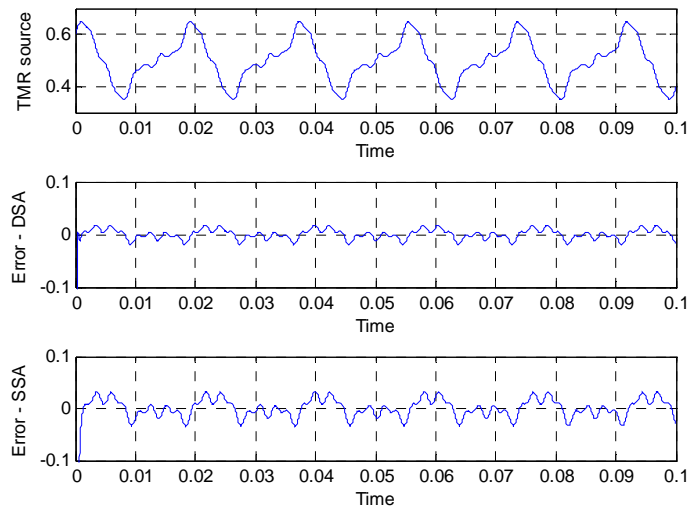

Fig. 5 Comparison of TMR disturbance rejection using modelreference SMC between DSA(dual-stage) and SSA(single-stage)
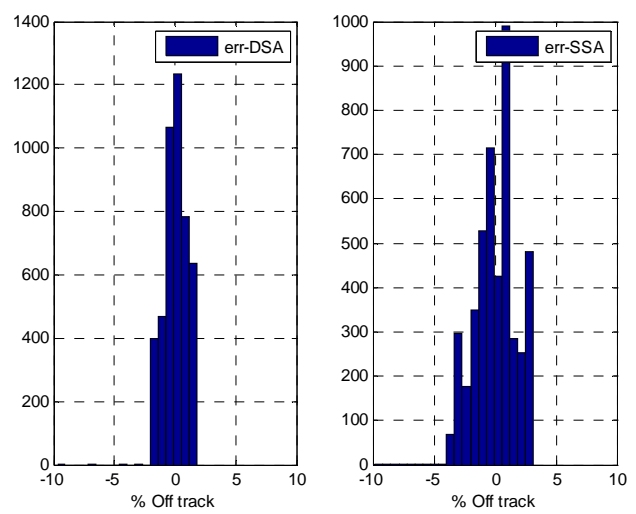

Fig. 6 Comparison of \%off track (PES) using model-reference SMC between DSA(dual-stage) and SSA(single-stage) 
The step responses of DSA with proposed model-reference SMC controller design is illustrated in Fig. 7 shows 5\% settling time is $0.35 \mathrm{msec}$. compared to SSA at $0.63 \mathrm{msec}$. The overshoot of the head displacement is less than $5 \%$ which is met per requirement specifications.

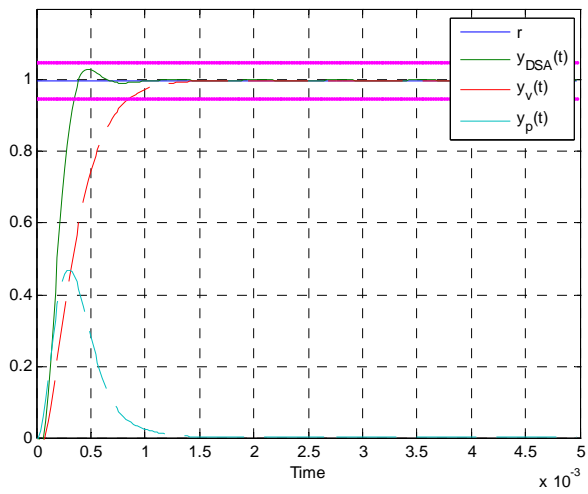

Fig. 7 Plot of response head displacement, VCM displacement and PZT displacement on DSA with model-reference SMC

\section{V.CONCLUSION}

In this paper model-reference SMC has provided robustness against plant model uncertainty and disturbances. The reference model is relating reference to the plant output, is formulated to meet various time-domain specifications. The $\mathrm{SMC}$ controller is designed and shown that the errors between the reference model state variables and the plant state variables are driven to zeros. As a result, the plant output is closed to the reference model output, and the specifications can be met. The performance of proposed controller has been verified through Matlab simulation and can be proven that the DSA servo control is a superior controller when compared to SSA. The method presented in this paper can also be applied to improve the performance of the dual-stage actuator even further.

\section{REFERENCES}

[1] Takashi Yamaguchi, "HDD Servo Control Development -Present and Future," SICE-ICASE International Joint Conference, October 2006

[2] K.C. Tan, et al., "Evolutionary design and implementation of a hard disk drive servo control system," Springer-Verlag, Published March 2006

[3] A. Al Mamun, T. H. Lee, and T. S. Low, "Frequency domain identification of transfer function model of a disk drive actuator," Mechatronics, vol. 12, pp. 563-574, May 2002.

[4] T. Suthasun, et al., "System identification and controller design for dual actuated hard disk drive," Control Engineering Practice 12 (2004)

[5] Bharat Bhushan (Ed.), Springer Handbook of Nanotechnology 2nd Edition. Springer 2005

[6] Chen BM, Lee TH, Venkataramanan V., Hard Disk Drives Servo Systems. Springer: New York, London, 2002

[7] Abdullah Al M., GuoXiao G, Chao Bi, Hard Disk Drive Mechatronics and Control. Taylor \& Francis, 2007

[8] H. Numasato and M. Tomizuka, "Settling control and performance of a dual-actuator system for hard disk drives," IEEE-ASME Transactions on Mechatronics, vol. 8, pp. 431-438, Dec 2003.

[9] Kemao Peng, et al., "Design and implementation of a dual-stage actuated HDD servo system via composite nonlinear control," Mechatronics 14 (2004) 965-988

[10] Zheng, Q. and M. Tomizuka., "Compensation of Dominant Frequency Components of Nonrepeatable Disturbance in Hard Disk Drives," IEEE Transactions on Magnetics. Vol. 43. No. 9. pp. 3756-3762. 2007
[11] You, K.H. and M. Hong, "Robust Linear Quadratic Sliding-Mode Control for Hard Disk Drives," IEEE Transactions on Instrumentation and Measurement. Vol. 56. No. 3. pp. 1087-1093. 2007

[12] Low, K.S. and T.S. Wong., "A Multiobjective Genetic Algorithm for Optimizing the Performance of Hard Disk Drive Motion Control System," IEEE Transactions on Industrial Electronics. Vol. 54. 2007

[13] Taghirad, H.D. and E. Jamei., "Robust Performance Verification of Adaptive Robust Controller for Hard Disk Drives," IEEE Transactions on Industrial Electronics. Vol. 55. No. 1. pp. 448-456. 2008.

[14] Du, C., et al., "Disturbance Rejection for a Data Storage System via Sensitivity Loop Shaping and Adaptive Nonlinear Compensation," IEEE/ASME Transactions on Mechatronics. Vol. 13. No. 5. 2008

[15] Roberto Horowitz, et al., "Dual-stage servo systems and vibration compensation in computer hard disk drive," Control Engineering Practice 15 (2007) 291-305

[16] Hongbo Zhang, et al., "Dual-stage HDD head positioning using an Hinf controller and a tracking differentiator," Mechatronics 19 (2009)

[17] Seung-Hi Lee, et al., "Design of A Dual-stage Actuator Control System with Discrete-Time Sliding Mode for Hard Disk Drives," IEEE Conference on Decision and Control, Australia, December 2000

[18] W.C. Wu, and T.S. Liu, "Sliding mode based learning control for trackfollowing in HDD," Mechatronics 14 (2004)

[19] Tsang-Li Tai, Jian-Shiang Chen, "Discrete-time sliding-mode controller for dual-stage systems -A hierarchical approach," Mechatronics 15 (2005) 949-967

[20] G. Herrmann, et al., "Application of a discrete sliding mode technique to a HDD dual-stage track-seek and track-following servo system," 22nd IEEE International Symposium on Intelligent Control (2007)

[21] Qinglei $\mathrm{Hu}$, et al., "Discrete-Time Sliding Mode Control With TimeVarying Surface for Hard Disk Drives," IEEE Trans Control System Technology, Vol. 17, No. 1, January 2009

[22] Edwards C, Spurgeon SK, Sliding Mode Control: Theory and Applications. Taylor \& Francis: U.K., 1998.

[23] Utkin, V. I., Sliding Modes in Control Optimization, Springer-Verlag, Berlin, 1992.

[24] Khalil, H. K., Nonlinear Systems, Prentice Hall, 2001. 